Ann. Biol. anim. Bioch. Biophys., 1979, 19 (3 B), 763-767.

\title{
Action comparée de divers composés glucidiques et azotés sur le transfert intestinal du calcium
}

\author{
par Sylviane TARDIVEL
}

EPHE, Physiologie-Métabolisme minéral des Mammifères, ERA-CNRS 070572

Faculté de Pharmacie 92290 Châtenay-Malabry.

Summary. The comparative action of various carbohydrate and nitrogenous compounds on infestinal calcium transfer.

Some carbohydrates increase calcium absorption probably because they are phosphate-acceptors. Can phosphorylatable nitrogen compounds also increase calcium transfer? When administered to adult rats, several nitrogenous compounds such as lysine, arginine creatine and creatinine increased calcium absorption, measured with a ${ }^{45} \mathrm{Ca}$ tracer. The activation of calcium absorption by L-lysine, as by carbohydrates, seemed to be saturated with high concentrations of that amino acid. All these molecules were more effective in the ileum than in the jejunum. L-lysine increased calcium transfer, if the terminal $\varepsilon \mathrm{NH}_{2}$ was ionized. Phosphorylated forms of arginine and creatine are known and the same terminal $\varepsilon \mathrm{NH}_{2}$ was phosphorylated in those molecules.

\section{Introduction.}

L'absorption intestinale du calcium est activée par certains composés glucidiques tels le sorbitol, l'arabinose, le xylose. Elle est inhibée par les phosphates (Fournier ef al., 1976a). L'activité d'une enzyme de la muqueuse intestinale, la phosphatase alcaline, est modulée de la même façon par ces mêmes effecteurs (Fournier et al., 1976b). La phosphatase alcaline est douée de propriétés transphosphorylantes. Elle pourrait donc jouer un rôle au niveau de l'absorption intestinale du calcium en phosphorylant le glucide, neutralisant ainsi l'effet inhibant des phosphates sur l'absorption du minéral. D'autres molécules non glucidiques phosphorylables, tels la choline et l'inositol, accroissent également le transfert intestinal du calcium (Fournier, 1955 ; Fournier, 1956). En 1956, Wasserman et al. montrent, chez le rat, que parmi 18 acides aminés, la lysine et l'arginine augmentent le transfert du calcium. Ces substances agiraient-elles sur l'absorption du calcium par un mécanisme identique à celui proposé pour les glucides? L'arginine-phosphate jouant dans le muscle des Invertébrés le même rôle que la créatine-phosphate chez les Vertébrés, la créatine ne serait-elle pas aussi active sur l'absorption du calcium? 


\section{Conditions expérimentales.}

Premier type d'expérience. - Deux $\mathrm{ml}$ de solution renfermant $20 \mu$ moles de $\mathrm{CaCl}_{2}$ et $0,2 \mu \mathrm{Ci}$ de ${ }^{45} \mathrm{Ca}$ et pouvant ou non contenir la substance azotée étudiée, sont administrés par intubation gastrique à des rats Wistar mâles de 5 mois, à jeun depuis $12 \mathrm{~h}$. Les animaux sont sacrifiés 24 h après l'intubation ; un fémur est prélevé et incinéré ; la radioactivité des cendres est déterminée. Un coefficient de rétention calcique fémorale est évalué de la manière suivante :

$$
\frac{{ }^{45} \mathrm{Ca} \text { du fémur }}{{ }^{45} \mathrm{Ca} \text { administré }} \times 1000 .
$$

Deuxième type d'expérience. - Un ml d'une solution de même composition que la précédente est injectée dans une anse ligaturée in sifu de l'intestin grêle. L'anse de $12 \mathrm{~cm}$ est faite au niveau jéjunal ou iléal. Deux heures après l'injection les animaux sont sacrifiés, l'anse ligaturée est prélevée ef incinérée ; la radioactivité des cendres est déterminée. Un coefficient d'absorption calcique est défini de la manière suivante :

$$
\frac{\left({ }^{45} \mathrm{Ca} \text { injecté }-{ }^{45} \mathrm{Ca} \text { de l'anse }\right) \times 100}{{ }^{45} \mathrm{Ca} \text { injecté }} .
$$

Diverses molécules azotées sont administrées selon les procédés expérimentaux précédemment décrits :

- la L-lysine, en anse intestinale, est donnée à la concentration $50 \mathrm{mM}$. Cette solution est injectée telle quelle, c'est-à-dire à $\mathrm{pH} 10,0$ ou après avoir été ajustée à $\mathrm{pH} 7,0$ par addition de $\mathrm{HCl}$. Elle est également administrée per os à concentrations croissantes $(12,5$ à $250 \mathrm{mM})$;

- la D-lysine est étudiée, en anse, à des concentrations de 100 ef $150 \mathrm{mM}$;

- arginine, créatine ef créatinine sont données en anses intestinales et per os aux concentrations respectives de 100,50 et $50 \mathrm{mM}$.

\section{Résultats.}

La figure 1 montre l'action de la L-lysine sur l'absorption du calcium, mesurée par la rétention fémorale. L'activation de cette rétention semble présenter un caractère saturable.

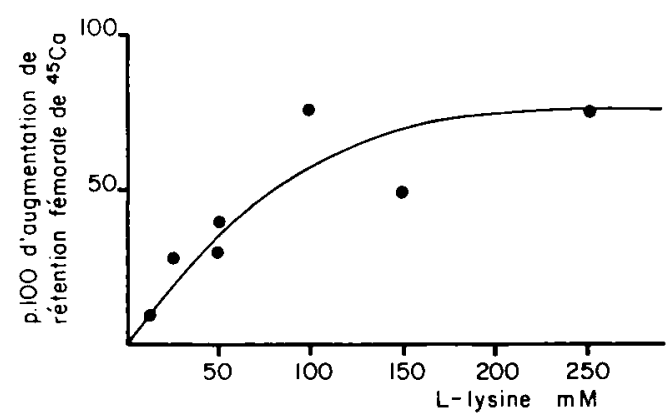

FIG. 1. - Effet de la L-lysine sur l'absorption de ${ }^{45} C a$ mesurée par la rétention fémorale. 
La L-lysine (50 mM) accroît le coefficient d'absorption du calcium de 53,8 p. 100 au niveau jéjunal, de 90,8 p. 100 au niveau iléal (tabl. 2), tandis que la D-lysine, dans l'iléum, accroît ce même coefficient de 92,5 p. 100 et de 137 p. 100 pour des concentrations respectives de 100 et $150 \mathrm{mM}$. Le $\mathrm{pH}$ des solutions de lysine doit être inférieur à 10 pour que la molécule soit active. $\mathrm{A}$ un $\mathrm{pH}$ de 10 , aucune action n'est observée.

La L-arginine (50 et $100 \mathrm{mM}$ ) augmente la rétention fémorale du calcium de 10,5 p. 100 et 31,5 p. 100 (tabl. 1) tandis que la créatinine se montre plus active à des concentrations équivalentes (tabl. 1).

\section{TABLEAU 1}

Action de divers composés azotés sur la rétention fémorale de ${ }^{45} \mathrm{Ca}$ administré per os

\begin{tabular}{lcccc}
\hline Molécule & $\begin{array}{c}\text { Concentration } \\
\mathrm{mM}\end{array}$ & $\begin{array}{c}{ }^{45} \mathrm{Ca} \text { du fémur } \\
\text { P. } 1000 \text { de la dose reçue per os }\end{array}$ & $\begin{array}{c}\text { p. } 100 \text { d'augmentation } \\
\text { par rapport au lot } 0\end{array}$ \\
\hline$\ldots \ldots \ldots \ldots$ & 0 & $11,05 \pm 1,10^{*}$ & & \\
L-lysine & 50 & $15,10 \pm 0,84$ & $\mathrm{P}<0,05 * *$ & 36,6 \\
& 100 & $19,46 \pm 0,50$ & $\mathbf{P}<0,001$ & 76,1 \\
L-arginine & 50 & $12,21 \pm 0,58$ & $\mathbf{p}<0,01$ & 10,5 \\
Créatine ..... & 100 & $14,53 \pm 2,30$ & $\mathrm{P}<0,05$ & 31,5 \\
Créatinine.... & 50 & $12,70 \pm 1,01$ & $\mathrm{NS}$ & 14,9 \\
& 50 & $15,90 \pm 1,39$ & $\mathrm{P}<0,001$ & 43,9 \\
\hline
\end{tabular}

*: $M \pm S M(n=6$ d̀ 25$)$.

** : p calculé selon le test de Student.

L'arginine n'a pu être étudiée en anse intestinale car elle entraîne des flux d'eau importants qui entravent la mesure d'un coefficient d'absorption. Créatine et créatinine, étudiées en anse intestinale, sont plus actives au niveau idéal qu'au niveau jéjunal (łabl. 2).

TABLEAU 2

Action de divers composés azofés sur l'absorption iléole ou jéjunale de ${ }^{45} \mathrm{Ca}$

\begin{tabular}{|c|c|c|c|c|c|}
\hline \multirow{2}{*}{$\begin{array}{l}\text { Molécule } \\
\text { concentration } \\
(50 \mathrm{mM})\end{array}$} & \multicolumn{5}{|c|}{ Coefficient d'absorption du ${ }^{45} \mathrm{Ca}$ en p. 100 de la dose reçue } \\
\hline & Jéjun & um & $\begin{array}{l}\text { P. } 100 \\
\text { d'augmen- } \\
\text { tation/lot } 0\end{array}$ & lléum & $\begin{array}{l}\text { P. } 100 \\
\text { d'augmen- } \\
\text { tation/lot } 0\end{array}$ \\
\hline 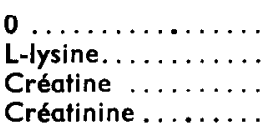 & $\begin{array}{l}29,44 \pm 1,82 * \\
45,30 \pm 3,10 \\
30,30 \pm 2,24 \\
34,92 \pm 1,03\end{array}$ & $\begin{array}{l}\mathrm{p}<0,01 * * \\
\text { NS } \\
\text { NS }\end{array}$ & $\begin{array}{r}53,8 \\
2,9 \\
18,6\end{array}$ & $\begin{array}{l}18,74 \pm 1,09 \\
35,76 \pm 5,41 p<0,001 \\
33,28 \pm 8,59 p<0,001 \\
29,18 \pm 3,18 p<0,01\end{array}$ & $\begin{array}{l}90,8 \\
77 \\
55,7\end{array}$ \\
\hline
\end{tabular}

* $M \pm S M(n=6$ à 25$)$;

** p calculé selon le test de Student. 


\section{Discussion.}

Plusieurs corrélations peuvent être établies entre l'action des glucides et des molécules azotées étudiées sur le transfert intestinal du calcium.

1) Comme pour les glucides, les diverses molécules aminées étudiées sont toujours plus actives dans l'iléum que dans le jéjunum.

2) L'activation présente un caractère saturable aussi bien pour les formes $D$ que les formes $L$.

3) Les composés naturels et non naturels se montrent aussi actifs (énantiomorphes de la lysine).

4) Arginine ef surtout créatine augmentent l'absorption du calcium. Toutes deux sont éminemment phosphorylables.

Nous nous sommes donc interrogés sur quelle partie de ces molécules pouvait porter la phosphorylation. Nous rappelons (fig. 2) les formules des différentes molécules étudiées.

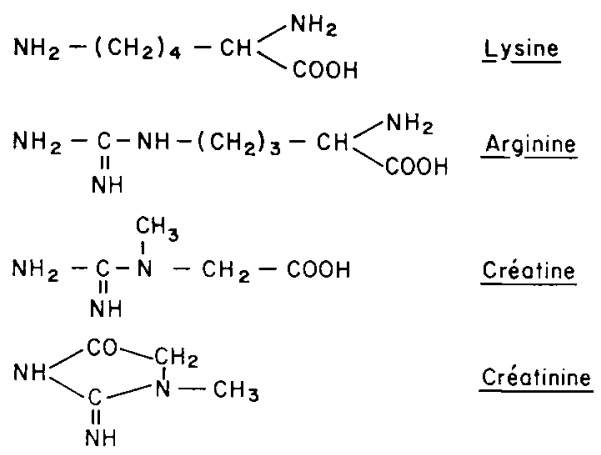

FIG. 2. - Formules des molécules étudiées.

Nous avons vu que la lysine n'était active, en anse intestinale, que si le $\mathrm{pH}$ de la solution était inférieur à 10 (voir chap. résultats). Dans ces conditions le $\varepsilon \mathrm{NH}_{2}$ de la molécule est ionisé. On peut supposer que ce même $\mathrm{NH}_{2}$ terminal que l'on retrouve dans toutes les molécules étudiées ou le - $\mathrm{NH}$ - de la liaison peptidique de la créatinine est la fonction à considérer. L'arginine et la créatine possèdent justement leurs dérivés phosphorylés en cette position.

De plus, on a pensé que l'effet inhibant des phosphates sur le transfert du calcium serait de précipiter le calcium sous forme de phosphate de calcium et ceci d'autant plus que le $\mathrm{pH}$ est alcalin. Cependant, le fait que l'on observe, en présence d'une solution très alcaline de lysine $(\mathrm{pH} 10)$, non pas la suppression de l'absorption calcique mais l'absorption de base que l'on observe en absence de l'acide aminé, semble indiquer qu'il n'y a pas d'insolubilisation quand la solution calcique administrée est à $\mathrm{pH}$ très alcalin. La lysine intervient donc autrement que par son action sur le $\mathrm{pH}$.

En résumé, diverses molécules : lysine, arginine, créatine, créatinine, certaines éminemment phosphorylables, accroissent l'absorption du calcium. Le but d'études 
ultérieures sera de mettre en évidence, de la même manière que cela a déjà été fait pour des composés glucidiques, nołamment le sorbitol, la formation de ces composés azotés phosphorylés au niveau de la muqueuse intestinale.

Commission CNERNA Digestion-Absorption/Association des Physiologistes, Paris 5-6 ocfobre 1978.

\section{References}

FOURNIER P., 1955. De l'action commune, ostéogène et lipotrope de la choline et du lactose. C.R. Acad. Sci. Paris, 241, 903-905.

FOURNIER P., 1956. Le pouvoir ostéogène du méso-inositol et la notion de substrat de croissance. C. R. Acad. Sci. Paris, 242, 1221-1224.

FOURNIER P., DUPUIS Y., FOURNIER A., 1976a. Etude, en anses intestinales in situ, de la modulation par un glucide et par un phosphate de l'absorption du calcium. C. R. Soc. Biol,, 170, 304-309.

FOURNIER P., DUPUIS Y., FOURNIER A., 1976b. Etude, au niveau de la muqueuse jéjunale, de effets de divers composés glucidiques sur l'activité de quelques enzymes localisées dans les bordures en brosse. Accroissement d'activité phosphatasique. C. R. Soc. Biol., 170, 739-744.

WASSERMAN R. H., COMAR C. L., NOLD M. M., 1956. The influence of amino-acids and other organic compounds on the gastrointestinal absorption of calcium and strontium in the rat. J. Nutr., 59, 371-383. 